\title{
EL PAPEL DE LAS FRACCIONES LIPÍDICAS, LIPOPROTEÍNAS Y APOLIPOPROTEÍNAS EN PREECLAMPSIA
}

\author{
Norma Cecilia SerRano Díaz, MD, MSC $C^{1}$, Sandra Milena AceVedo, $M^{2}$ \\ María Teresa Otero Motta, $M^{3}$, Claudia Carolina Colmenares Mejía, MD $^{1}$ \\ Melisa Tatiana SePÚllVEda Jaime ${ }^{4}$, Edgar Humberto Forero GaLVIS ${ }^{4}$ \\ Wiston Alberto Rodríguez Rocha ${ }^{4}$, María Carolina PÁez Leal, MD MSc ${ }^{1}$ \\ ${ }^{1}$ Centro de Investigaciones Biomédicas, Universidad Autónoma de Bucaramanga, Bucaramanga, Colombia. \\ ${ }^{2}$ Residente Primer Año, Medicina Interna UNAB. \\ ${ }^{3}$ Servicio de Urgencias. Clínica Carlos Ardila Lulle. \\ ${ }^{4}$ Estudiante programa de Medicina, Facultad de Ciencias de la Salud. Universidad Autónoma de Bucaramanga.
}

\begin{abstract}
Resumen
La Preeclampsia (PE) es un trastorno multisistémico, exclusivo de la gestación humana y responsable de aproximadamente 44.000 muertes maternas anuales a nivel mundial; esta enfermedad está asociada a múltiples complicaciones tanto en la madre como en el feto, sin embargo, su etiología no se encuentra totalmente dilucidada y en consecuencia ha sido difícil definir estrategias válidas de predicción. La PE es una enfermedad compleja y comparte diferentes factores de riesgo con las enfermedades cardiovasculares; como la obesidad, hipertensión arterial, resistencia a la insulina y dislipidemias. Con esto se evidencia que algunas alteraciones en los niveles lípidos y apolipoproteínas, se asocian con mayor peroxidación lipídica y estrés oxidativo lo cual puede desencadenar en disfunción endotelial para ambas patologías. Por tanto, la exploración de la evidencia de una asociación entre las fracciones lipídicas y riesgo de PE, puede aportar nuevo conocimiento en torno a la etiología de esta enfermedad. En la presente revisión, se plantearán las principales implicaciones biológicas de las alteraciones del perfil lipídico y apolipoproteínas en la génesis de la PE. Se describirán los estudios observacionales que se han aproximado a su evaluación y se identificarán sus principales debilidades metodológicas, con el fin de plantear estrategias para una evaluación integral de esta vía fisiopatológica, con posibles implicaciones predictivas de la enfermedad.
\end{abstract}

Palabras clave: Preeclampsia, dislipidemias, apolipoproteínas, peroxidación lipídica, análisis de randomización mendeliana.

\section{THE ROLE OF LIPID FRACTIONS, LIPOPROTEINS AND APOLIPOPROTEINS IN PREECLAMPSIA}

\begin{abstract}
Preeclampsia (PE) is a multisystemic disorder unique to human pregnancy and responsible for about 44,000 maternal deaths worldwide. This disease is associated with multiple complications for both mother and fetus; however, its etiology is not totally clear and it has therefore been difficult to define valid prediction strategies. PE is a complex disease and it shares different risk factors with cardiovascular disease, such as obesity, hypertension, insulin resistance and dyslipidemia, evidencing that the presence of disturbances in lipid and apolipoprotein levels are associated with increased lipid peroxidation
\end{abstract}

* Correspondencia: Dra. Páez, Centro de Investigaciones Biomédicas UNAB. Calle 157 N 19 - 55 Cañaveral Parque, Bucaramanga, Colombia. E-mail: mpaez@unab.edu.co. PBX: 6399155, ext:505, Fax: 6399147. 
and oxidative stress which can lead to endothelial dysfunction in both diseases. Therefore, exploring the evidence of an association between lipid fractions and PE risk can provide new knowledge about the etiology of this disease. In this review, the major biological implications of lipid profile and apolipoproteins alterations in the genesis of PE will be presented, describing the observational studies and identifying its main methodological weaknesses in order to create strategies for comprehensive assessment of the pathophysiological pathway with potential predictive implications of the disease.

Key words: Preeclampsia, Dyslipidemias, apolipoproteins, lipid peroxidation, Mendelian Randomization Analysis.

\title{
O PAPEL DAS FRAÇÕES LIPÍDICAS, LIPOPROTEÍNAS E APOLIPOPROTEÍNAS NA PREECLAMPSIA
}

\section{Resumo}

\begin{abstract}
A Preeclampsia (PE) é um transtorno multissistêmico, exclusivo da gestação humana e responsável por aproximadamente 44.000 mortes maternas anuais a nível mundial; esta doença esta associada a múltiplas complicações tanto na mãe como no feto, porém, sua etiologia não está totalmente esclarecida e consequentemente tem sido difícil definir estratégias válidas de predição. A PE é uma doença complexa e compartilha diferentes fatores de risco com as doenças cardiovasculares, como a obesidade, hipertensão arterial, resistência à insulina e dislipidemias; evidenciando que alterações nos níveis dos lipídios e apolipoproteínas, se associam com maior peroxidação lipídica e estresse oxidativo que pode desencadear disfunção endotelial para ambas patologias. Portanto, a exploração da evidência de uma associação entre las frações lipídicas e risco de PE, pode contribuir com novo conhecimento ao redor da etiologia desta doença. Na presente revisão, serão apresentadas as principais implicações biológicas das alterações do perfil lipídico e apolipoproteínas na gênese da $\mathrm{PE}$, descrevendo os estudos observacionais que se aproximaram à sua avaliação $e$ identificando suas principais fraquezas metodológicas, com o objetivo de propor estratégias para uma avaliação integral desta via fisiopatológica, com possíveis implicações preditivas da doença.
\end{abstract}

Palavras chave: Preeclampsia, dislipidemias, apolipoproteínas, peroxidação lipídica, análise de randomização mendeliana.

La Preeclampsia (PE) es un trastorno multisistémico exclusivo de la gestación humana, con una incidencia global entreel 5-7\% en todos los embarazos (1),y es responsable de aproximadamente 44.000 muertes maternas anuales en el mundo (2). En Colombia, la PE para el año 2008 fue la primera causa de mortalidad materna, con una tasa estimada de 46 por cada 100.000 nacidos vivos (2). Si bien, en las últimas tres décadas se ha logrado disminuir el número de muertes maternas por esta afección, actualmente sigue representando un problema importante en salud pública, que afecta el binomio madre-hijo. La PE causa múltiples complicaciones tanto en la madre como en el feto, lo cual ha generado un interés creciente en investigación. Sin embargo, aún se desconoce su etiología y en consecuencia no ha sido fácil definir estrategias válidas de predicción y manejo.
La PE comparte factores de riesgo convencionales con las Enfermedades Cardiovasculares (ECV), tales como, la obesidad, dislipidemias, hipertensión arterial y resistencia a la insulina (3-5). La base común a estos trastornos, es la presencia de disfunción endotelial, la cual se asocia con el desarrollo de vasoespasmo, aumento de la permeabilidad vascular y estímulo de la agregación plaquetaria (6), predisponiendo a disfunción placentaria y aterosclerosis, ambos hallazgos característicos de la PE y otras ECV.

Una de las vías asociadas con la disfunción endotelial, se relaciona con la presencia de alteraciones en los niveles plasmáticos de lípidos y apolipoproteínas, como fuente de peroxidación lipídica y estrés oxidativo (7). Algunos estudios han realizado aproximaciones al respecto, describiendo en suero o placenta el incremento 
de los radicales libres de oxígeno y la disminución de sustancias antioxidantes, que conlleva a un desequilibrio generador de daño permanente de la célula endotelial (8).Sin embargo, estos resultados parecen no ser concluyentes y su implicación directa no ha sido claramente definida. En la presente revisión, se plantearán las principales implicaciones biológicas de las alteraciones del perfil lipídico y apolipoproteínas en la génesis de la PE, describiendo estudios observacionales que se han aproximado a su evaluación $e$ identificando sus principales debilidades metodológicas, con el objetivo de plantear estrategias para una evaluación integral de esta vía fisiopatológica, con posibles implicaciones predictivas.

\section{Preeclampsia como Enfermedad Cardiovascular: alteraciones en las fracciones lipídicas y apolipoproteínas como una vía fisiopatológica común}

La PE se caracteriza por una respuesta vascular anormal a la placentación, la cual produce una respuesta sistémica dada por un incremento en la resistencia vascular periférica, activación del sistema de la coagulación, aumento de la agregación plaquetaria, estrés oxidativo, peroxidación lipídica, disfunción endotelial y producción anormal de factores de crecimiento vascular $(9,10)$. Hallazgos similares, se encuentran en ECV, como enfermedad coronaria y cerebrovascular (11). Es por esto, que recientemente, ha venido emergiendo evidencia que sugiere a la PE como un desorden cardiovascular, que comparte muchos de los factores de riesgo convencionales ya definidos para las $\mathrm{ECV}$, tales como presión arterial elevada, obesidad, resistencia a la insulina, sedentarismo y dislipidemia (12). Está por evaluar si también comparten factores de riesgo no convencionales, genéticos, ya descritos para ambas condiciones.

La relación entre las fracciones lipídicas y el riesgo de ECV ha sido evaluada de forma robusta, especialmente el papel de los triglicéridos, colesterol LDL y colesterol HDL (13-15). El Prospective Studies Collaboration que incluyó datos individuales de 61 estudios prospectivos con cerca de 55.000 muertes de origen vascular, encontró que una disminución de $38.6 \mathrm{mg} / \mathrm{dL}$ de colesterol total, representaba una disminución del riesgo de enfermedad isquémica cardíaca en todos los grupos de edad evaluados. Sin embargo, se encontró que la relación colesterol total/ HDL es un $40 \%$ mejor predictor de muerte de origen coronario en relación con valores aislados de HDL y dos veces más que el colesterol total (16).

Con base en estos resultados y tomando en cuenta las similitudes de la PE con los desórdenes cardiovasculares, varios investigadores han desarrollado más de 25 estudios observacionales (rango de las muestras analizadas entre 7 a 173 casos), para evaluar el papel de los lípidos en PE. En la Tabla 1, se resumen los hallazgos de los principales estudios observacionales realizados en mujeres gestantes, los cuales son sugestivos de un incremento en el riesgo de la PE asociado con niveles elevados de colesterol LDL, triglicéridos y bajos niveles de colesterol HDL. Algunos estudios reportan incremento en el riesgo de PE de hasta 3.6 veces en mujeres gestantes con niveles elevados de colesterol LDL y disminución del colesterol HDL (17-20).

Tabla 1. Características generales de los estudios observacionales realizados a la fecha que cuantifican perfil lipídico en Preeclampsia.

\begin{tabular}{|l|l|l|l|l|c|c|c|}
\hline Autor (Referencia) & Año & \multicolumn{1}{|c|}{ Diseño } & \multicolumn{1}{|c|}{ País } & \multicolumn{1}{c|}{ Etnicidad } & $\begin{array}{c}\text { Total } \\
\text { casos }\end{array}$ & $\begin{array}{c}\text { Total } \\
\text { controles }\end{array}$ & $\begin{array}{c}\text { Total } \\
\text { muestra }\end{array}$ \\
\hline Rodie VA (21) & 2004 & Casos y Controles & UK & Blanca & 23 & 23 & 46 \\
\hline Barden AE (22) & 1999 & Cohorte & Australia & Blanca & 62 & 84 & 146 \\
\hline Wakatsuki A (23) & 2000 & Casos y Controles & Japón & Asiática & 12 & 12 & 24 \\
\hline Raijmakers MT (24) & 2004 & Casos y Controles & Holanda & Blanca & 40 & 40 & 80 \\
\hline Vanderjagt DJ (25) & 2004 & Casos y Controles & Nigeria & Negra & 43 & 130 & 173 \\
\hline Ware-Jauregui S (26) & 1999 & Casos y Controles & Perú & Hispana & 125 & 179 & 304 \\
\hline Powers RW (27) & 1998 & Casos y Controles & Pensilvania & Mezclada & 21 & 33 & 54 \\
\hline Enquobahrie DA (17) & 2004 & Cohorte & USA & Blanca & 57 & 510 & 567 \\
\hline Vadachkoria S (28) & 2006 & Casos y Controles & Zimbabwe & Negra & 170 & 184 & 354 \\
\hline Clausen T (29) & 2001 & Cohorte & Noruega & Blanca & 71 & 2086 & 2157 \\
\hline
\end{tabular}




\begin{tabular}{|l|c|l|l|l|c|c|c|}
\hline Kaaja R (30) & 1995 & Casos y Controles & Finlandia & Blanca & 31 & 21 & 52 \\
\hline Maseki M (31) & 1981 & Casos y Controles & Japón & Asiática & 23 & 22 & 45 \\
\hline Gratacos E (32) & 1996 & Cohorte & España & Mezclada & 115 & 115 & 230 \\
\hline Williams M (33) & 2003 & Casos y Controles & Zimbabwe & Negra & 173 & 186 & 359 \\
\hline Murai J (34) & 1997 & Casos y Controles & USA & Mezclada & 31 & 31 & 62 \\
\hline Baker A (35) & 2009 & Casos y Controles & USA & Negra & 50 & 100 & 150 \\
\hline Mayret-Mesquiti M (36) & 2007 & Casos y Controles & México & Hispana & 27 & 47 & 74 FranzH(37) \\
\hline Llurbaa E (38) & 2005 & Casos y Controles & España & Mezclada & 34 & 23 & 57 \\
\hline Ogura K (39) & 2002 & Cohorte & Japón & Asiática & 7 & 19 & 26 \\
\hline Hubel C (40) & 1998 & Casos y Controles & UK & Blanca & 20 & 20 & 40 \\
\hline Catarino C (41) & 2008 & Casos y Controles & Portugal & Mezclada & 46 & 42 & 88 \\
\hline Yamaguchi K (42) & 1988 & Casos y Controles & Japón & Asiática & 29 & 16 & 45 \\
\hline Sánchez SE (43) & 2003 & Casos y Controles & Perú & Hispana & 113 & 150 & 263 \\
\hline Mikhail MS (44) & 1995 & Casos y Controles & USA & Mezclada & 29 & 46 & 75 \\
\hline Autor (Referencia) & Criterio & PAS & Criterio PAD & $\begin{array}{l}\text { Criterio } \\
\text { Proteinuria }\end{array}$ & $\begin{array}{l}\text { Tiempo de toma } \\
\text { de muestra * }\end{array}$ & Marcadores** & \\
\hline
\end{tabular}

Tabla 2. Principales hallazgos de los estudios observacionales realizados a la fecha que cuantifican perfil lipídico en preeclampsia

\begin{tabular}{|c|c|c|c|c|c|c|}
\hline \multirow[t]{2}{*}{ Autor (Referencia) } & \multicolumn{3}{|c|}{ Criterios definitorios de PE } & \multirow[t]{2}{*}{$\begin{array}{c}\text { Tiempo de toma } \\
\text { de muestra * }\end{array}$} & \multirow[t]{2}{*}{ Marcadores** } & \multirow[t]{2}{*}{ Asociación } \\
\hline & PAS & PAD & Proteinuria & & & \\
\hline Rodie VA (21) & . & 90 & $300 \mathrm{mg} / 24 \mathrm{~h}, 2+$ & 3 & PLC & si \\
\hline Barden AE (22) & 140 & 90 & $300 \mathrm{mg} / 24$ & 3 & PLC & si \\
\hline Wakatsuki A (23) & 160 & 110 & $\geq 30 \mathrm{mg} / \mathrm{dl}$ & 3 & PLC & si \\
\hline Raijmakers MT (24) & . & 90 & $300 \mathrm{mg} / 24$ & 3,4 & PLC & si \\
\hline Vanderjagt DJ (25) & 140 & 90 & 190 mg x cada gr de creatinina & 3 & PLC & si \\
\hline Ware-Jauregui S (26) & 140 & 90 & $>30 \mathrm{mg} / \mathrm{dl}, 1+$ & 3 & PLC & si \\
\hline Powers RW (27) & 140 & 90 & $>500 \mathrm{mg} / 24-\mathrm{h}$ & 2 & TGS & No \\
\hline Enquobahrie DA (17) & 140 & 90 & $>30 \mathrm{mg} / \mathrm{dl}, 1+$ & 1 & PLC & $\mathrm{Si}$ \\
\hline Vadachkoria S (28) & 140 & 90 & $>30 \mathrm{mg} / \mathrm{dl}, 1+$ & 4 & TGS & $\mathrm{Si}$ \\
\hline Clausen T (29) & 140 & 90 & $>300 \mathrm{mg} / \mathrm{dl}, 1+$ & 2 & PLC & $\mathrm{Si}$ \\
\hline Kaaja R (30) & 140 & 90 & $>0.5 \mathrm{~g} / \mathrm{dia}$ & 3 & PLC & $\mathrm{Si}$ \\
\hline Maseki M (31) & . & . & . & 3 & PLC & $\mathrm{Si}$ \\
\hline Gratacos E (32) & 140 & 90 & $>300 \mathrm{mg} / \mathrm{L}$ en $24 \mathrm{~h}$ & $1,2,3$ & C-Total - TGS & $\mathrm{Si}$ \\
\hline Williams M (33) & 140 & 90 & $>30 \mathrm{mg} / \mathrm{dl}, 1+$ & 4 & PLC & $\mathrm{Si}$ \\
\hline Murai J (34) & 140 & 90 & $>30 \mathrm{mg} / \mathrm{dl}, 1+$ & 3 & TGS & $\mathrm{Si}$ \\
\hline Baker A (35) & 140 & 90 & $\geq 300 \mathrm{mg} / 24 \mathrm{~h}$ & 2 & PLC & $\mathrm{Si}$ \\
\hline Mayret-Mesquiti M (36) & 140 & 90 & $\geq 30 \mathrm{mg} / \mathrm{dL}$ & 3 & PLC & $\mathrm{Si}$ \\
\hline Franz H (37) & . & 90 & . & . & PLC & No \\
\hline Llurbaa E (38) & . & 90 & $300 \mathrm{mg} / 24 \mathrm{~h}, 2+$ & 3 & PLC & $\mathrm{Si}$ \\
\hline Ogura K (39) & 140 & 90 & $\geq 300 \mathrm{mg} / 24 \mathrm{~h}$ & $1,2,3,4$ & PLC & $\mathrm{Si}$ \\
\hline Hubel C (40) & 140 & 90 & $\geq 500 \mathrm{mg} / 24 \mathrm{~h}$ & 3 & PLC & $\mathrm{Si}$ \\
\hline
\end{tabular}




\begin{tabular}{|c|c|c|c|c|c|c|}
\hline \multirow[t]{2}{*}{ Autor (Referencia) } & \multicolumn{3}{|c|}{ Criterios definitorios de PE } & \multirow{2}{*}{$\begin{array}{c}\text { Tiempo de toma } \\
\text { de muestra * }\end{array}$} & \multirow[t]{2}{*}{ Marcadores** } & \multirow[t]{2}{*}{ Asociación } \\
\hline & PAS & PAD & Proteinuria & & & \\
\hline Catarino C (41) & & 90 & $>30 \mathrm{mg} / \mathrm{dl}, 1+$ & 3 & PLC & $\mathrm{Si}$ \\
\hline Yamaguchi K (42) & & & . & 3 & PLC & No \\
\hline Sanchez SE (43) & . & . & . & 3 & TGS & $\mathrm{Si}$ \\
\hline Mikhail MS (44) & & . & . & 3 & PLC & $\mathrm{Si}$ \\
\hline
\end{tabular}

* Tiempo de toma: 1,2 y 3 primer, segundo y tercer trimestre, 4:puerperio.

** PLC: Perfil Lipídico Completo, triacilgliceroles.

La evidencia de una asociación entre fracciones lipídicas y riesgo de $\mathrm{PE}$ no solo proviene de estudios en mujeres gestantes (casos y controles o cohortes), también se observa cuando los niveles de lípidos se cuantifican en mujeres antes del embarazo, como recientemente fue demostrado por Magnussen et al. en un estudio prospectivo en Noruega (45). Los investigadores evaluaron los niveles de las fracciones lipídicas antes de la gestación en 3.494 mujeres (133 casos incidentes de PE), y demostraron que niveles altos de C-total y de C-LDL se asocian con un incremento en el riesgo de PE con un OR-ajustado de 2,1 (IC95\% 1,2 - 3,8) y 2,4 (IC95\% 1,3 - 4,3) respectivamente, efecto que al parecer es gradual y monotónico. Por otra parte, no se encontró relación con bajos niveles de HDL y altos niveles de triacilgliceroles al realizar un ajuste por posibles variables de confusión. Sin embargo, se evidenció una tendencia al incremento en el riesgo con un PE OR-ajustado 1,3 (IC95\% 0,8 - 2,4) y 1,6 (IC95\% 0,9 - 2,9), respectivamente (45).

En la misma vía, las apolipoproteínas intervienen en la unión de las lipoproteínas a sus receptores celulares, facilitando su captación. La Apolipoproteína A (Apo A) es el principal componente polipeptídico de las HDL y tiene dos formas principales Apo A-I, la cual constituye el 75\% de la Apo A en las HDL, y la Apo A-II, la cual constituye el 25\% de las HDL. La Apo $\mathrm{B}$, en cambio,es el principal componente de las LDL (80\%) y del 40\% de las VLDL.

Estudios observacionales postulan valores de referencia para Apo A-I entre 115-224 mg/dl y de Apo-B entre $60-130 \mathrm{mg} / \mathrm{dL}$ (46). Estos valores de referencia no se pueden extrapolar a las mujeres gestantes, quienes presentan cambios importantes en el metabolismo lipídico y en particular las pacientes preeclámpticas. Si tenemos en cuenta, que la PE es una Enfermedad Compleja, en la cual los factores genéticos interactúan de manera compleja con los factores medioambientales, es pertinente citar estudios que han evaluado la asociación de las Apo en $\mathrm{PE}$ desde ambas perspectivas.

Al revisar los estudios observacionales que han evaluado Apo en PE, se encuentran 19 publicados (Tabla 2). La mayoría de los estudios fueron realizados en Europa $(73,7 \%)$ y Asia (21,05\%). No ha sido publicado a la fecha ningún estudio conducido en América Latina y África, a pesar de ser los continentes con mayor tasa de mortalidad materna asociada a trastornos hipertensivos. El 95\% de los estudios eran de casos y controles, que incluían mujeres durante el tercer trimestre de gestación con un tamaño muestral promedio de 72 gestantes, 29,3 casos y 42 controles. Los marcadores evaluados con mayor frecuencia son Apo B (15 estudios) y Apo A-I (14 estudios), encontrándose solo tres trabajos para Apo E, donde la literatura ha estado más dirigida a estudios de asociación genética.

Desde la perspectiva genética el mejor modelo es la ApoE. Esta molécula, es un componente importante de las lipoproteínas de muy baja densidad VLDL, encargada de modificar respuestas inflamatorias y retirar el exceso de colesterol de la circulación, mediante la regulación de la absorción de lípidos en el hígado (50). Su gen, APOE, ubicado en 19q13.2, tiene tres alelos comunes, que codifican para tres isoformas de ApoE en el plasma (ApoE2, ApoE3 y ApoE4), dos de las cuales (ApoE2 y ApoE4) han sido asociadas con niveles altos de triglicéridos y VLDL. Por tanto, se postula que los polimorfismos del gen APOE están asociados a un incremento del riesgo de ECV y PE. Nagy et al., encontraron una frecuencia mayor del alelo ApoE2 en mujeres con PE que en controles, hallazgo que no ha sido reproducible $(62,63)$. 
A pesar de la evidencia sugestiva de una asociación entre lípidos, apolipoproteínas y PE, esta evidencia proviene de estudios con un tamaño de muestra pequeño tanto para casos como para controles, y un análisis limitado de las posibles variables de confusión, condiciones que aumentan la posibilidad de generar asociaciones espurias, sesgos o falso positivos como posibles explicaciones de estos resultados y su falta de reproducibilidad. Adicionalmente, la gran mayoría de los estudios se han realizado en población de origen caucásico, por lo tanto, queda sin resolver el interrogante sí esta asociación tiene validez para poblaciones con diferente grado de mestizaje, como la colombiana.

Tabla 3. Características generales de los estudios observacionales que cuantifican apolipoproteínas (Apo A-I, ApoB, ApoE) en preeclampsia durante el tercer trimestre de embarazo.

\begin{tabular}{|c|c|c|c|c|c|c|c|c|c|c|}
\hline $\begin{array}{c}\text { Autor } \\
\text { (Referencia) }\end{array}$ & Año & $\begin{array}{c}\text { Diseño } \\
\text { del estudio }\end{array}$ & País & Etnicidad & $\begin{array}{l}\text { Total } \\
\text { Casos }\end{array}$ & $\begin{array}{c}\text { Total } \\
\text { Controles }\end{array}$ & $\begin{array}{c}\text { Total } \\
\text { muestra }\end{array}$ & \multicolumn{3}{|c|}{ Marcadores } \\
\hline & & & & & & & & A-I & B & $\mathbf{E}$ \\
\hline Bayhan G (19) & 2005 & Casos y Controles & Turquía & Blanca & 20 & 25 & 45 & $\mathrm{X}$ & $\mathrm{X}$ & \\
\hline Kaaja (30) & 1995 & Casos y Controles & Finlandia & Blanca & 31 & 21 & 52 & $\mathrm{X}$ & $\mathrm{X}$ & \\
\hline Hubel CA (40) & 1999 & Casos y Controles & USA & Mezclada & 20 & 20 & 40 & & $\mathrm{X}$ & \\
\hline Catarino C (41) & 2008 & Casos y Controles & Portugal & NR & 46 & 42 & 88 & $\mathrm{X}$ & $\mathrm{X}$ & \\
\hline Zhang (47) & 2011 & Casos y Controles & China & Asiática & 16 & 16 & 32 & $\mathrm{X}$ & & \\
\hline Kocyigit (48) & 2004 & Cohorte & Turquía & Blanca & 45 & 30 & 85 & $\mathrm{X}$ & $\mathrm{X}$ & \\
\hline Belo L (49) & 2004 & Casos y Controles & Portugal & NR & 51 & 144 & 195 & $\mathrm{X}$ & & \\
\hline $\operatorname{Var} A(50)$ & 2003 & Casos y Controles & Turquía & Blanca & 30 & 30 & 60 & $\mathrm{X}$ & $\mathrm{X}$ & \\
\hline Hayman RG (51) & 1999 & Casos y Controles & Inglaterra & Blanca & 10 & 10 & 20 & $\mathrm{X}$ & $\mathrm{X}$ & \\
\hline Kobayashi (52) & 1992 & Casos y Controles & Tokio & Asiática & 22 & 12 & 34 & $\mathrm{X}$ & $\mathrm{X}$ & $\mathrm{X}$ \\
\hline Rosing U (53) & 1989 & Casos y Controles & Suiza & Blanca & 26 & 21 & 47 & $\mathrm{X}$ & & \\
\hline Demir B (54) & 2011 & Casos y Controles & Turquía & Blanca & 35 & 35 & 70 & $\mathrm{X}$ & $\mathrm{X}$ & \\
\hline Lei Q (55) & 2011 & Casos y Controles & China & Asiática & 33 & 200 & 233 & & $\mathrm{X}$ & \\
\hline Sanrandol (56) & 2004 & Casos y Controles & Turquía & Blanca & 42 & 20 & 62 & $\mathrm{X}$ & $\mathrm{X}$ & \\
\hline Cekmen (57) & 2003 & Casos y Controles & Turquía & Blanca & 34 & 32 & 66 & $\mathrm{X}$ & $\mathrm{X}$ & \\
\hline Winkler K (58) & 2003 & Casos y Controles & Alemania & Blanca & 15 & 23 & 38 & & $\mathrm{X}$ & \\
\hline Bai H (59) & 2002 & Casos y Controles & China & Asiática & 31 & 24 & 55 & $\mathrm{X}$ & $\mathrm{X}$ & $\mathrm{X}$ \\
\hline Bartha JL (60) & 1999 & Casos y Controles & España & NR & 10 & 17 & 27 & & $\mathrm{X}$ & \\
\hline Chalas J (61) & 2002 & Casos y Controles & Francia & Blanca & 24 & 25 & 49 & & & $\mathrm{X}$ \\
\hline
\end{tabular}

$\mathrm{Al}$ analizar los resultados serológicos, encontramos que para Apo A-I, el $\sim 43 \%$ de los estudios lo describe como un factor protector para PE $(47-49,53,54)$, mientras que los restantes no corroboraron esta asociación $(50,51,56,57)$; comportamiento que es similar para ApoB, donde seis estudios $(40,41,52,56,57,60)$ lo identifican como un factor de riesgo para PE, pero los hallazgos no son reproducibles en los demás trabajos $(19,20,50,51,30,54,55,59)$. Inconsistencias muy similares a las demostradas previamente para los estudios en fracciones lipídicas.

\section{Implicaciones biológicas de la dislipidemia y el estrés oxidativo en Preeclampsia}

La disfunción endotelial desempeña un papel importante en la patogénesis de la PE, explicando las principales características clínicas de esta entidad, tales como la hipertensión arterial, proteinuria, edema y activación de sistema de la coagulación. Existen dos vías que podrían conducir a disfunción endotelial: la primera, por medio de sustancias que produce el tejido decidual y son liberadas hacia la circulación materna. La segunda, la coexistencia de condiciones maternas 
que predispongan a su presentación, como: hipertensión esencial, diabetes mellitus tipo 2, dislipidemia, resistencia a la insulina y adiposidad.

En el primer caso, factor decidual, se conoce que la transformación limitada de las arterias espirales lleva a estrés oxidativo en la circulación uteroplacentaria, dando como resultado la producción de derivados de la oxidación lipídica, generándose un desequilibrio entre pro y anti-oxidantes (64). Los lípidos peroxidados, además de generar daño local, se unen a las lipoproteínas circulantes y son ampliamente distribuidos causando daño oxidativo sistémico $(65,66)$. Algunos autores sugieren que este estrés oxidativo conlleva a la activación de leucocitos maternos y posteriormente, activación endotelial, acúmulo de ácidos grasos y colesterol en la íntima y, en general un desbalance oxidativo con las repercusiones claramente conocidasde este fenómeno (65).

Los ácidos grasos poliinsaturados interactúan con los radicales libres y son fuente de peroxidación lipídica en la placenta y el suero materno en gestantes con PE. Dichos lípidos peroxidados producen efectos deletéreos sobre el endotelio in vitro y pueden producir disfunción endotelial, exacerbado por la reducción en la síntesis de prostaciclina. Los ácidos grasos inducen acumulación de triglicéridos en las células endoteliales cultivadas, y la resistencia a la insulina secundaria al daño endotelial, puede explicarse parcialmente por los niveles de triglicéridos elevados (Figura 1) (69).

\section{Perioxidación lipídica}

Radicales libres + Ácidos grasos $\rightarrow$ Triglicéridos oxidados, LDL oxidado
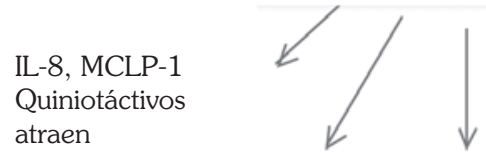

Lesión endotelial (tono y permeabilidad)

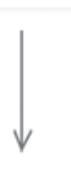

Rp scavenger Macrófago:

Célula espumosa

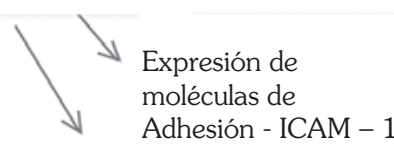

Proliferación y migración de células musculares lisas al espacio subendotelial

\section{Antioxidantes exógenos y endógenos}

\author{
Glutation reductasa \\ Superoxido Dismutasa \\ Vitamina E \\ Vitamina C \\ B-carotenos
}

Factores del troboblasto

Factores maternos
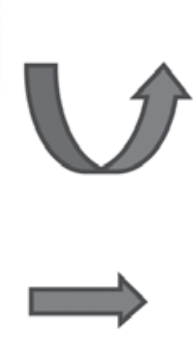

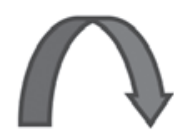

Implantación normal y desarrollo anormal

Perfusión de oxígeno

Placentaria alterada

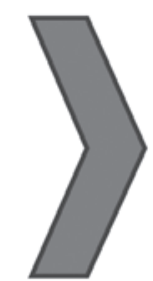

Inactividad física

Estrés oxidativo

Dislipidemia

Dieta pobre

Resistencia a la insulina

Figura 1. Patogénesis de la Preeclampsia explicada por el incremento de la peroxidación lipídica.

En concordancia con lo descrito, las arterias espirales frecuentemente presentan depósitos de colesterol y fosfolípidos, un fenómeno denominado 'aterosis aguda', y en la decidua de mujeres con PE además muestra un alto contenido de lípidos peroxidados y formas libres de isoprostano 8-iso, marcador lipídico de estrés oxidativo (70). Por todo lo anterior, la decidua puede ser otra fuente de derivados de la peroxidación lipídica, que cuando alcanzan la circulación sistémica materna, inducen disfunción endotelial. 
Un mecanismo potencial para la activación endotelial vascular mediada por los productos de la peroxidación lipídica, está relacionado con la activación del factor nuclear de transcripción kappa B (NF $\mathrm{kB}$, por sus siglas en inglés). Una vez activado el $\mathrm{NF} k \mathrm{kB}$ se une a los elementos cis-reguladores del DNA $e$ induce la expresión de varias citocinas pro inflamatorias como la proteína quimiotáctica de monocitos tipo 1 (MCP-1) y la interleukina-8 (IL-8) y moléculas de adhesión celular, como las ICAM-1. La activación de monocitos circulantes por parte de las citocinasproinflamatorias junto con la expresión endotelial creciente de ICAM-1, da lugar a la activación de numerosos macrófagos asociados a lesiones ateroscleróticas $(69,70)$.

El embarazo normal se asocia a niveles elevados de especies reactivas de oxígeno y peróxidos de los lípidos a nivel placentario, sin embargo, mujeres condislipidemia, producen mayores niveles de peróxidos lipídicos, haciendo que la capacidad de los sistemas antioxidantes de neutralización placentariase sature y se instaure un estado patológico de tensión oxidativa, similar al observado en gestantes con PE (71).

\section{Las apolipoproteínas son predictores más fuertes de riesgo cardiovascular, que la relación LDL/HDL. Su papel en la Preeclampsia aún está por esclarecer}

Evidencia derivada del estudio multi-centrico INTERHEART (15.000 casos y 15.000 controles) ha sugerido que las Apo A-I y B (y la relación ApoB/Apo A-I) tienen mayor efecto predictivo que las LDL para identificación de individuos en alto riesgo de eventos vasculares (72).

Como se describió anteriormente, las Apo también han sido implicadas en PE de una manera similar a la ECV, no obstante, el grado de evidencia no es tan fuerte como el observado con las fracciones lipídicas, TGL, y LDL $(19,20)$.

Desde la perspectiva de la prevención primordial las variantes alélicas de APOE "E2" y "E4", han sido fuertemente asociadas con un incremento en el riesgo de enfermedad coronaria (73). Se ha comprobado un claro efecto sobre los niveles de ApoE de una forma que reproduce la asociación sobre riesgo coronario, evidencia que hace de la ApoE un excelente candidato a ser evaluado en PE.

\section{Limitaciones de la evidencia sobre el posible papel causal de los lípidos y apolipoproteínas en Preeclampsia}

A pesar del gran número de estudios funcionales, desarrollados en animales de experimentación o en modelos moleculares y de estudios observacionales que indican la presencia de una asociación; el posible papel causal de los lípidos y las apolipoproteínas en $\mathrm{PE}$, presenta importantes limitaciones que merecen ser descritas en detalle. Si la asociación es real, tendría un gran impacto en términos de salud pública sí consideramos la posibilidad de una intervención terapéutica para reducir el riesgo de $\mathrm{PE}$, y por ende su impacto en el binomio madre-hijo. Las principales limitaciones que identificamos en los estudios observacionales son:

1. El reducido tamaño de muestra (Tabla 1 y 2) de los estudios conducidos hasta el momento, en promedio 40 casos por estudio, lo cual podría significar que las diferencias en los niveles sean solo falsos positivos, por una probabilidad de error tipo I mayor a 5\%, máxime si se tiene en cuenta la baja reproducibilidad de estos resultados.

2. La presencia de sesgo de causalidad reversa, hace que la enfermedad desde sus estadios tempranos lleve a incrementos en los niveles de lípidos y lipoproteínas y conduzca a la presencia de asociación de éstos con PE $(74,75)$. Es decir, los niveles de lípidos incrementados no serían la causa, sino una consecuencia de la PE, y la asociación se debería a una inversión temporal de los sucesos, interpretándose el efecto como la causa.

3. Debido a que las concentraciones de lípidos, lipoproteínas y apolipoproteínas se correlacionan fuertemente con otros factores de riesgo para PE (v.g. obesidad, diabetes, hipertensión arterial y nutricionales) (72), se genera la posibilidad de una asociación positiva de estos marcadores con PE debido a confusión residual (76). Esto es importante si se tiene en cuenta que en la mayoría de los estudios, los ajustes por posibles variables de confusión son insuficientes. 
4. La presencia de heterogeneidad clínica, representada por unos criterios definitorios de PE no unificados y en algunos reportes muy ambiguos, implicaría el ingreso de "casos" de pacientes no diagnosticadas adecuadamente y generaría dilución del efecto. De manera complementaria, la inadecuada selección de controles, aumentan la probabilidad de generar asociaciones espurias.

5. Se ha detectado de manera sistemática un análisis estadístico limitado, por una parte, debido a que los estudios observacionales, poseen poca claridad en los criterios de exclusión, que al no ser tan rigurosos, no controlan todas las variables dependientes. Y por otra parte, la realización de múltiples subgrupos de análisis, que aumenta significativamente el error tipo I y que amerita estrategias de análisis particulares, por ejemplo, corregir a través de múltiples comparaciones, las cuales por lo general no son implementadas (77).

\section{Randomización Mendeliana:estrategiaalternativa para evaluar el papel causal de los lípidos, lipoproteínas y apolipoproteínas en Preeclampsia. Aproximación del Estudio GenPE}

La randomización mendeliana aparece como una estrategia alternativa para controlar por la presencia de factores de confusión y sesgo de causalidad reversa. El objetivo, va dirigido a identificar una variante genética que se asocie de forma consistente con diferencias en los fenotipos intermedios, para nuestro caso, con las concentraciones de circulantes lípidos, o lipoproteínas o apolipoproteínas. Lo anterior se basa en la segunda Ley de Mendel, que implica que el heredar o no una variante genética es un proceso aleatorio que ocurre al momento de la formación del gameto, independiente de cualquier factor de confusión conocido o desconocido, esto es una perfecta equivalencia entre los estudios genéticos de asociación de tamaño adecuado y los ensayos clínicos controlados (78). Por lo tanto, si altas concentraciones de, por ejemplo, Apo Eson causal en el desarrollo de PE, el ser portador de un genotipo que expone a las mujeres a altas concentraciones de Apo $\mathrm{E}(\mathrm{M} / \mathrm{M})$ a largo plazo y de manera constante, debería incrementar el riesgo de PE en una manera proporcional a las diferencias en los niveles de Apo $\mathrm{E}(\triangle \mathrm{ApoEs})$ atribuibles a la presencia de dicho genotipo (Figura 2). Esta relación, debería estar en gran medida libre de confusión y de sesgo de causalidad reversa $(79,80)$. Aún más, debido a que el genotipo es una característica fija en el tiempo, el sesgo de verificación (recall bias) es eliminado (57) y el sesgo de la regresión de la dilución, es poco probable que ocurra; pues su presencia es resultado de errores de medición y variabilidad biológica de la exposición, que lleva a subestimar la asociación entre un factor de riesgo y la enfermedad (81).

La estrategia de randomización mendeliana $(82,83)$, se ha usado recientemente para estudiar el vínculo entre homocisteína y ECV $(84,85)$, así como también para evaluar el efecto de la proteína C-reactiva en la enfermedad arterial coronaria y en la DMT2 (86-88).

El estudio de casos y controles GenPE (Genética y Preeclampsia), con cerca de 9.000 pacientes colombianas captadas desde el año 2000 a la fecha (www. genpe.org). Éste se convierte en un escenario natural para evaluar por medio de randomización mendeliana el poder de asociación entre las fracciones lipídicas (colesterol total, colesterol-no HDL), lipoproteínas (LDL, HDL) y apolipoproteínas (Apo A-I, Apo B, Apo E) y $\mathrm{PE}$, en nuestra población. Para dar respuesta a esta hipótesis, se ha realizado la cuantificación de estos biomarcadores en 2.000 gestantes con PE y 2.000 controles, captados en Cartagena, Cali, Medellín, Bogotá y Bucaramanga. Una vez se realicen estas cuantificaciones en la totalidad de la muestra seleccionada, los resultados serán usados en conjunto con los hallazgos del GWAS (por su siglas en ingles: Genome-Wide Association Studies) adelantado por nuestro grupo (http://www.wtccc.org.uk/ccc2/ projects/ccc2_pa.shtml), para realizar el análisis de Randomización Mendeliana y determinar así, el papel causal de las fracciones lipídicas y apolipoproteínas en PE.

\section{Agradecimiento}

Departamento Administrativo de Ciencia, Tecnología e Innovación (Colciencias, contrato: 505-2009) y Universidad Autónoma de Bucaramanga - UNAB (Proyecto EGEN52). 


\title{
Fenotipo Intermedio
}

(Niveles séricos ApoEs

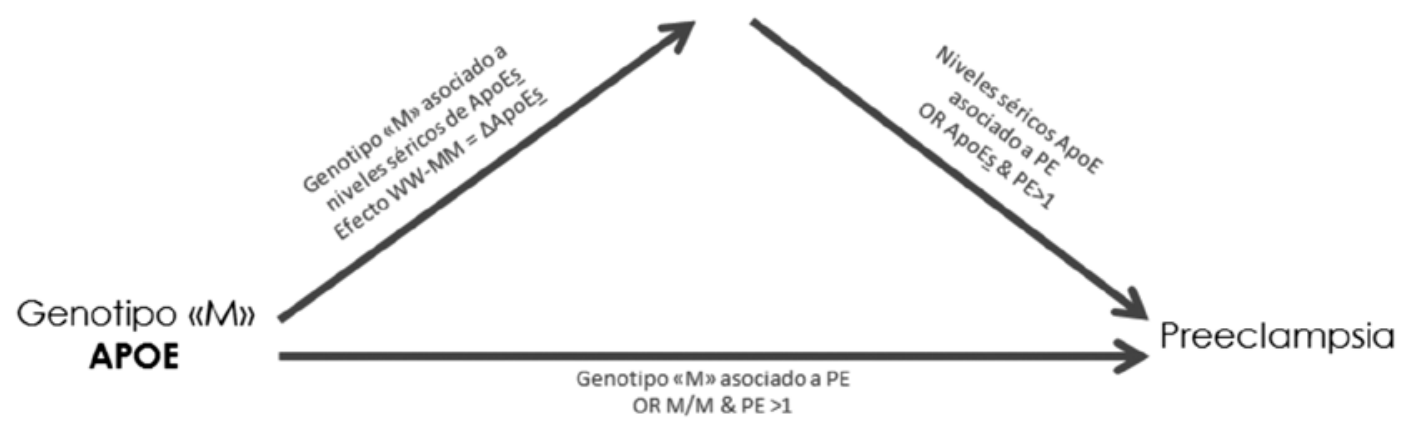

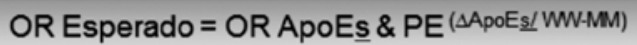

\begin{abstract}
MM: Genotipo asociado a altos niveles de ApoE en suero
WW: Wild Type, Genotipo silvestre asociado a bajos niveles de ApoE en suero
\end{abstract}

Figura 2. Estrategia para determinar el papel causal de las apolipoproteínas en Preeclampsia.

\section{Referencias bibliográficas}

1. World Health Organization International Collaborative Study of Hypertensive Disorders of Pregnancy. Geographic Variation in the incidence of hypertension in pregnancy. Am J Obstet Gynecol. 1998; 158(1): 80-83.

2. Khan K, Wojdyla D, Say L, Gülmezoglu A, Van Look P. WHO analysis of causes of maternal death: a systematic review. Lancet. 2006; 367(9516):1066-1074.

3. Garovic V, Baileyb K, Boerwinklec E, Huntd S, Wedere A, et al. Hypertension in pregnancy as a risk factor for cardiovascular disease later in life. J Hypertens. 2010; 28(4): 826-833.

4. Girouard J, Giguère Y, Moutquin J, and Forest J. Previous Hypertensive Disease of Pregnancy Is Associated With Alterations of Markers of Insulin Resistance. Hypertension. 2007; 49(5): 1056-1062.

5. Roberts J, Gammill H. Insulin Resistance in Preeclampsia. Hypertension. 2006; 47(3): 341-342.

6. Poston L. Endothelial dysfunction in pre-eclampsia. Pharmacol Rep. 2006; 58Suppl: 69-74.

7. Zavalza-Gómez AB. Obesity and oxidative stress: a direct link to preeclampsia? Arch Gynecol Obstet. 2011; 283(3): 415-422.

8. Redman CWG, Sargent IL. Placental debris, oxidative stress and preeclampsia. Placenta.2000; 21(7): 597-602.

9. Sibai B, Dekker G, Kupferminc M. Pre-eclampsia. Lancet. 2005; 365(9461): 785-799.

10. Savvidou MD, Hingorani AD, Tsikas D, Frolich JC, Vallance $\mathrm{P}$, Nicolaides $\mathrm{KH}$. Endothelial dysfunction and raised plasma concentrations of asymmetric dimethylarginine in pregnant women who subsequently develop pre-eclampsia. Lancet. 2003; 361(9368): 1511-1517.

11. Murphy BP, Stanton T, Dunn FG. Hypertension and myocardial ischemia. Med Clin North Am. 2009; 93(3): 681-695.
12. Cnossen JS, Leeflang MM, de Haan EE, Mol BW, van der Post JA, Khan KS, et al. Accuracy of body mass index in predicting pre-eclampsia: bivariate meta-analysis. BJOG. 2007; 114(12): 1477-1485.

13. Berg LH. Lipid measurements for coronary risk assessment: a review. Am J Med Technol. 1981; 47(7): 539-543.

14. Francis KT. HDL cholesterol and coronary heart disease. South Med J. 1980; 73(2): 169-173.

15. Davignon J, Gregg RE, Sing CF. Apolipoprotein E polymorphism and atherosclerosis. Arteriosclerosis. 1988; 8(1): 1-21.

16. Prospective Studies Collaboration, Lewington S, Whitlock G, Clarke R, Sherliker P, Emberson J, Halsey J, et al. Blood cholesterol and vascular mortality by age, sex, and blood pressure: a meta-analysis of individual data from 61 prospective studies with 55,000 vascular deaths. Lancet. 2007; 372(9635): 1829-1839.

17. Enquobahrie DA, Williams MA, Butler CL, Frederick IO, Miller RS, Luthy DA. Maternal plasma lipid concentrations in early pregnancy and risk of preeclampsia. Am J Hypertens. 2004; 17(7): 574-581.

18. Belo L, Caslake M, Gaffney D, Santos-Silva A, Pereira-Leite L, Quintanilha A, et al. Changes in LDL size and HDL concentration in normal and preeclamptic pregnancies. Atherosclerosis. 2002; 162(2): 425-432.

19. Bayhan G, Koçyigit Y, Atamer A, Atamer Y, Akkus Z. Potential atherogenic roles of lipids, lipoprotein(a) and lipid peroxidation in preeclampsia. GynecolEndocrinol. 2005; 21(1): 1-6.

20. Koçyigit Y, Atamer Y, Atamer A, Tuzcu A, Akkus Z. Changes in serum levels of leptin, cytokines and lipoprotein in preeclamptic and normotensive pregnant women. GynecolEndocrinol. 2004;19(5): 267-273.

21. Rodie VA, Caslake MJ, Stewart F, Sattar N, Ramsay JE, Greer IA, Freeman DJ. Fetal cord plasma lipoprotein status in uncomplicated human pregnancies and in pregnancies com- 
plicated by pre-eclampsia and intrauterine growth restriction. Atherosclerosis. 2004; 176(1): 181-187.

22. Barden AE, Beilin LJ, Ritchie J, Walters BN, Michael C. Does a predisposition to the metabolic syndrome sensitize women to develop pre-eclampsia? J Hypertens. 1999; 17(9): 1307-1315.

23. Wakatsuki A, Ikenoue N, Okatani Y, Shinohara K, Fukaya T. Lipoprotein particles in preeclampsia: susceptibility to oxidative modification. Obstet Gynecol. 2000; 96(1): 55-59.

24. Raijmakers MT, van Tits BJ, Hak-Lemmers HL, Roes EM, Steegers EA, Peters WH. Low plasma levels of oxidized low density lipoprotein in preeclampsia. Acta Obstet Gynecol Scand. 2004; 83(12): 1173-1177.

25. Vanderjagt DJ, Patel RJ, El-Nafaty AU, Melah GS, Crossey MJ, Glew RH. High-density lipoprotein and homocysteine levels correlate inversely in preeclamptic women in northern Nigeria. ActaObstetGynecol Scand. 2004; 83(6): 536-542.

26. Ware-Jauregui S, Sanchez SE, Zhang C, Laraburre G, King $\mathrm{IB}$, Williams MA. Plasma lipid concentrations in pre-eclamptic and normotensive Peruvian women. Int J Gynaecol Obstet. 1999; 67(3): 147-155.

27. Powers RW, Evans RW, Majors AK, Ojimba JI, Ness RB, Crombleholme WR, Roberts JM. Plasma homocysteine concentration is increased in preeclampsia and is associated with evidence of endothelial activation. Am J Obstet Gynecol. 1998; 179(6 PT 1): 1605-1611.

28. Vadachkoria S, Woelk GB, Mahomed K, Qiu C, Muy-Rivera M, Malinow MR, Williams MA. Elevated soluble vascular cell adhesion molecule-1, elevated Homocyst(e)inemia, and hypertriglyceridemia in relation to preeclampsia risk. Am J Hypertens. 2006; 19(3): 235-242.

29. Clausen T, Djurovic S, Henriksen T. Dyslipidemia in early second trimester is mainly a feature of women with early onset pre-eclampsia. BJOG. 2001; 108(10): 1081-1087.

30. Kaaja R, Tikkanen MJ, Viinikka L, Ylikorkala O. Serum lipoproteins, insulin, and urinary prostanoid metabolites in normal and hypertensive pregnant women. Obstet Gynecol. 1995; 85(3): 353-356.

31. Maseki M, Nishigaki I, Hagihara M, Tomoda Y, Yagi K. Lipid peroxide levels and lipids content of serum lipoprotein fractions of pregnant subjects with or without pre-eclampsia. ClinChimActa. 1981; 115(2): 155-161.

32. Gratacós E, Casals E, Sanllehy C, Cararach V, Alonso PL, Fortuny A. Variation in lipid levels during pregnancy in women with different types of hypertension. ActaObstetGynecol Scand. 1996; 75(10):896-901.

33. Williams MA, Woelk GB, King IB, Jenkins L, Mahomed K. Plasma carotenoids, retinol, tocopherols, and lipoproteins in preeclamptic and normotensive pregnant Zimbabwean women. Am J Hypertens. 2003; 16(8):665-672.

34. Murai JT, Muzykanskiy E, Taylor RN. Maternal and fetal modulators of lipid metabolism correlate with the development of preeclampsia. Metabolism. 1997; 46(8):963-967.

35. Baker AM, Klein RL, Moss KL, Haeri S, Boggess K. Maternal serum dyslipidemia occurs early in pregnancy in women with mild but not severe preeclampsia. Am J Obstet Gynecol. 2009; 201(3): 293.e1-4.

36. Mayret-Mesquiti M, Pérez-Méndez O, Rodríguez ME, Fortoul TI, Gorocica P, Bernal-Alcántara D, Montaño LF, AlvaradoVasquez N. Hypertriglyceridemia is linked to reduced nitric oxide synthesis in women with hypertensive disorders of pregnancy. Hypertens Pregnancy. 2007; 26(4): 423-431.

37. Franz H, Wendler D. A controlled study of maternal serum concentrations of lipoproteins in pregnancy-induced hypertension. Arch Gynecol Obstet. 1992; 252(2): 81-86.
38. Llurba E, Casals E, Domínguez C, Delgado J, Mercadé I, Crispi F, Martín-Gallán P, Cabero L, Gratacós E. Atherogenic lipoprotein subfraction profile in preeclamptic women with and without high triglycerides: different pathophysiologic subsets in preeclampsia. Metabolism. 2005; 54(11): 1504-1509.

39. Oqura K, Miyatake T, Fukui O, Nakamura T, Kameda T, Yoshino G. Low-density lipoprotein particle diameter in normal pregnancy and preeclampsia. J AtherosclerThromb. 2002; 9(1): 42-47.

40. Hubel CA, Lyall F, Weissfeld L, Gandley RE, Roberts JM. Small low-density lipoproteins and vascular cell adhesion molecule-1 are increased in association with hyperlipidemia in preeclampsia. Metabolism. 1998; 47(10): 1281-1288.

41. Catarino C, Rebelo I, Belo L, Rocha-Pereira P, Rocha S, Castro EB, Patrício B, Quintanilha A, Santos-Silva A. Fetal lipoprotein changes in pre-eclampsia. Acta Obstet Gynecol Scand. 2008; 87(6): 628-634.

42. Yamaguchi K. Triglycerides and apoproteins in toxemia of pregnancy. Nihon SankaFujinkaGakkaiZasshi. 1988; 40(12): 1875-1882.

43. Sanchez SE, Zhang C, Williams MA. The influence of maternal triglyceride levels on infant birth weight in Peruvian women with pre-eclampsia. J Matern Fetal Neonatal Med. 2003; 13(5): 328-333.

44. Mikhail MS, Basu J, Palan PR, Furgiuele J, Romney SL, Anyaegbunam A. Lipid profile in women with preeclampsia:relationship between plasma triglyceride levels and severity of preeclampsia. J Assoc Acad Minor Phys. 1995; 6(1): 43-45.

45. Magnussen EB, Vatten LJ, Lund-Nilsen TI, Salvesen KA, Davey Smith G, Romundstad PR. Prepregnancy cardiovascular risk factors as predictors of pre-eclampsia: population based cohort study. BMJ. 2007; 10; 335(7627): 978.

46. McQueen MJ, Hawken S, Wang X, Ounpuu S, Sniderman A, Probstfield J, Steyn K, Sanderson JE, Hasani M, Volkova E, Kazmi K, Yusuf S; INTERHEART study investigators. Lipids, lipoproteins, and apolipoproteins as risk markers of myocardial infarction in 52 countries (the INTERHEART study): a case-control study. Lancet. 2008 19; 372(9634): 224-233.

47. Zhang H, Zhang Y, Yang F, Li L, Liu S, Xu Z, Wang J, Sun S. Complement component C4A and apolipoprotein A-I in plasmas as biomarkers of the severe, early-onset preeclampsia. Mol Biosyst. 2011; 7(8): 2470-2479.

48. Koçyigit Y, Atamer Y, Atamer A, Tuzcu A, Akkus Z. Changes in serum levels of leptin, cytokines and lipoprotein in preeclamptic and normotensive pregnant women. Gynecol Endocrinol. 2004; 19(5): 267-273.

49. Belo L, Gaffney D, Caslake M, Santos-Silva A, Pereira-Leite L, Quintanilha A, Rebelo I. Apolipoprotein E and cholesteryl ester transfer protein polymorphisms in normal and preeclamptic pregnancies. Eur J Obstet Gynecol Reprod Biol. 2004; 112(1): 9-15.

50. Var A, Kucu NK, Koyuncu F, Uyanik BS, Onur E, Yildirim Y, Oruç S. Atherogenic profile in preeclampsia. Arch Gynecol Obstet. 2003; 268(1): 45-47.

51. Hayman RG, Sattar N, Warren AY, Greer I, Johnson IR, Baker PN. Relationship between myometrial resistance artery behavior and circulating lipid composition. Am J Obstet Gynecol. 1999; 180(2 Pt 1): 381-386.

52. Kobayashi S, Tanaka M, Masaki K, Hirakawa S, Momose K. Apolipoprotein levels in preeclamptic pregnancies. Nihon SankaFujinkaGakkaiZasshi. 1992; 44(2): 223-228.

53. Rosing U, Samsioe G, Olund A, Johansson B, Kallner A. Serum levels of apolipoprotein A-I, A-II and HDL-cholesterol in second half of normal pregnancy and in pregnancy complicated by pre-eclampsia. HormMetab Res. 1989; 21(7):376-382. 
54. Demir B, Demir S, Atamer Y, Guven S, Atamer A, Kocyigit Y, Hekimoglu A, Toprak G. Serum levels of lipids, lipoproteins and paraoxonase activity in pre-eclampsia. $\mathrm{J}$ Int Med Res. 2011; 39(4): 1427-1431.

55. Lei Q, Lv LJ, Zhang BY, Wen JY, Liu GC, Lin XH, Niu JM. Ante-partum and post-partum markers of metabolic syndrome in pre-eclampsia. J Hum Hypertens. 2011; 25(1): 11-17.

56. Sarandöl E, Safak O, Dirican M, Uncu G. Oxidizability of apolipoprotein B-containing lipoproteins and serum paraoxonase/ arylesterase activities in preeclampsia. ClinBiochem. 2004; 37(11): 990-996.

57. Cekmen MB, Erbagci AB, Balat A, Duman C, Maral H, Ergen K, Ozden M, Balat O, Kuskay S. Plasma lipid and lipoprotein concentrations in pregnancy induced hypertension. ClinBiochem. 2003; 36(7): 575-578.

58. Winkler K, Wetzka B, Hoffmann MM, Friedrich I, Kinner M, Baumstark MW, Zahradnik HP, Wieland H, März W. Triglyceride-rich lipoproteins are associated with hypertension in preeclampsia. J ClinEndocrinolMetab. 2003; 88(3): 1162-1666.

59. Bai H, Liu X, Liu R, Liu Y, Li M, Liu B. Analysis of serum lipid and apolipoprotein levels in pregnancy-induced hypertension and normotensive pregnant women. Hua Xi Yi Ke Da XueXueBao. 2002; 33(1): 58-61.

60. Bartha JL, Comino-Delgado R, Bedoya FJ, Barahona M, Lubian D, Garcia-Benasach F. Maternal serum nitric oxide levels associated with biochemical and clinical parameters in hypertension in pregnancy. Eur J ObstetGynecolReprod Biol. 1999; 82(2): 201-207.

61. Chalas J, Audibert F, Francoual J, Le Bihan B, Frydman R, Lindenbaum A. Concentrations of apolipoproteins E, C2, and C3 and lipid profile in preeclampsia. Hypertens Pregnancy. 2002; 21(3): 199-204.

62. Carty D, Delles C, Dominiczak A. Novel Biomarkers for Predicting Preeclampsia. Trends Cardiovasc Med. 2008; 18(5): 186-194.

63. Nagy B, Rigo J, Fintor L, Romics L, Papp Z, et al. Distribution of Apolipoprotein(a) Isoforms in Normotensive and Severe Preeclamptic Women. J Matern Fetal Med 1999; 8(6):270-274

64. Henriksen T. The role of lipid oxidation and lipid oxidative derivatives in the development of preeclampsia. SeminPerinatol. 2000; 24(1): 29-32.

65. Atamer Y, Koçyigit Y, Yokus B, Atamer A, Erden AC. Lipid peroxidation, antioxidant defense, status of trace metals and leptin levels in preeclampsia. Eur J ObstetGynecolReprod Biol. 2005; 119(1):60-66.

66. Raijmakers MT, Peters WH, Steegers EA, Poston L. NAD(P)H oxidase associated superoxide production in human placenta from normotensive and pre-eclamptic women. Placenta. 2004; Suppl A: S85-89.

67. Dowhan W, Bodanov H. Functional roles of lipids in membranes. Biochemistry of Lipids, Lipoproteins and Membranes, 4th ed. Vance DE, Vance JE, editors. Elsevier; 2002.

68. Murray RK, Granner DK, Rodwell VW. Lipids of physiologic significance. Harper's illustrated biochemistry. 27th ed. Papadakis MA, Mcphee SJ,editores. McGraw-Hill Companies, Inc.; 2006.

69. Gratacos E, Asals E, Nllehy A, Cararach V, Alonso P et al. Variation in lipid levels during pregnancy in women with different types of hypertension. ActaObsteGynecol Scand 1996; 75(10): 896-901.
70. Takacs P, Kauma S, Sholley M, Walsh S, Dinsmoor J , Green $\mathrm{K}$. Increased circulating lipid peroxides in severe preeclampsia activate NF- $\mathrm{kB}$ and upregulate ICAM-1 in vascular endothelial cells. The FASEB. 2001; 15(2):279-281.

71. Hubel C. Oxidative stress in pathogenesis of preeclampsia. Proc Soc Exp Biol Med. 1999;222(3): 222-235.

72. McQueen MJ, Hawken S, Wang X, Ounpuu S, Sniderman A, Probstfield J. Lipids, lipoproteins, and apolipoproteins as risk markers of myocardial infarction in 52 countries (the INTERHEART study): a case-control study. Lancet. 2008; 372(9634): 224-233

73. Bennet AM, Di Angelantonio E, Ye Z, Wensley F, Dahlin A, Ahlbom A, et al. Association of apolipoprotein E genotypes with lipid levels and coronary risk. JAMA. 2007; 298(11) 1300-1311.

74. Davey Smith G, Ebrahim S. 'Mendelian randomization': can genetic epidemiology contribute to understanding environmental determinants of disease? Int J Epidemiol. 2003; 32(1): 1-22

75. Keavney B. Genetic epidemiological studies of coronary heart disease. Int J Epidemiol. 2002; 31(4):730-736.

76. Smith GD, Phillips AN. Confounding in epidemiological studies: why "independent" effects may not be all they seem. BMJ. 1992; 305(6836): 757-759.

77. Bellamy L, Casas JP, Hingorani AD, Williams DJ. Preeclampsia and the risk of cardiovascular disease and cancer in later life: a systematic review and meta-analysis. BMJ. 2007; 335(7627): 974

78. Morgan TH. Heredity and sex. New York:Columbia University Press; 1913: 27.

79. Davey Smith G, Ebrahim S. 'Mendelian randomization': can genetic epidemiology contribute to understanding environmental determinants of disease? Int J Epidemiol. 2003; 32(1):1-22.

80. Keavney B. Genetic epidemiological studies of coronary heart disease. Int J Epidemiol. 2002; 31(4): 730-736.

81. Colhoun HM, McKeigue PM, Davey Smith G. Problems of reporting genetic associations with complex outcomes. Lancet 2003; 361(9360): 865-872

82. Smith GD, Ebrahim S. Mendelian randomization: prospects, potentials, and limitations. Int J Epidemiol 2004; 33(1): 30-42.

83. Hingorani A, Humphries S. Nature's randomised trials. Lancet. 2005; 366(9501): 1906-1908.

84. Casas JP, Bautista LE, Smeeth L, Sharma P, Hingorani AD. Homocysteine and stroke: evidence on a causal link from mendelian randomization. Lancet 2005; 365(9455): 224-232.

85. Klerk M, Verhoef P, Clarke R, Blom HJ, Kok FJ, Schouten EG; MTHFR Studies Collaboration Group. MTHFR 677C--> T polymorphism and risk of coronary heart disease: a metaanalysis. JAMA 2002; 288(16): 2023-2031.

86. Casas JP, Shah T, Cooper J, Hawe E, McMahon AD, Gaffney $\mathrm{D}$ et al. Insight into the nature of the CRP-coronary event association using Mendelian randomization. Int $\mathrm{J}$ Epidemiol. 2006; 35(4): 922-931.

87. Hingorani AD, Shah T, Casas JP. Linking observational and genetic approaches to determine the role of $\mathrm{C}$-reactive protein in heart disease risk. Eur Heart J. 2006; 27(11):1331-1337.

88. Brunner EJ, Kivimäki M, Witte DR, Lawlor DA, Davey Smith G, Cooper JA, et al. Inflammation, insulin resistance, and diabetes - Mendelian randomization using CRP haplotypes points upstream. PLoS Med. 2008; 12(5): e155. 